\title{
Genotyping-by-Sequencing to Predict Resistance to Lima Bean Downy Mildew in a Diversity Panel
}

\author{
T. T. Mhora, E. G. Ernest, R. J. Wisser, T. A. Evans, M. E. Patzoldt, N. F. Gregory, S. E. Westhafer, \\ S. W. Polson, and N. M. Donofrio
}

First, third, fourth, fifth, sixth, seventh, and ninth authors: Department of Plant and Soil Sciences, University of Delaware, Newark; second author: University of Delaware Research and Education Center, Georgetown; and eighth author: Center for Bioinformatics and Computational Biology, University of Delaware, Newark.

Accepted for publication 28 May 2016.

\begin{abstract}
Mhora, T. T., Ernest, E. G., Wisser, R J., Evans, T. A., Patzoldt, M. E., Gregory, N. F., Westhafer, S. E., Polson, S. W., and Donofrio, N. M. 2016. Genotyping-by-sequencing to predict resistance to lima bean downy mildew in a diversity panel. Phytopathology 106:1152-1158.

Lima bean is affected by severe downy mildew epidemics caused by the oomycete Phytophthora phaseoli. There are six documented races of P. phaseoli (A to F). Race F is currently predominant in the mid-Atlantic region, creating the need for resistant lima bean cultivars with desirable agronomic characteristics. In order to develop markers for detecting race $\mathrm{F}$ resistance, bulked segregant analysis (BSA) using genotypingby-sequencing (GBS) was used on a biparental $\mathrm{F}_{2}$ population comprised of 216 lima bean progeny segregating for a dominant race $\mathrm{F}$ resistance

phenotype. Data were analyzed using a custom bioinformatic analysis pipeline (redrep). Kompetitive allele-specific polymerase chain reaction assays were developed using 12 GBS markers associated with the race $F$ resistance phenotype. Using these assays, the $F_{2}$ population was used to map the race $\mathrm{F}$ resistance locus. Seven markers were in linkage and significantly associated with race $F$ resistance that mapped between two markers located approximately 4.88 centimorgan $(\mathrm{cM})$ apart. These assays were successfully used to genotype a newly acquired lima bean diversity panel consisting of 256 landraces, cultivars, and wild germplasm, and a haplotype consisting of two of the seven linked markers was demonstrated to accurately predict race $\mathrm{F}$ resistance. This confirmed the ability of our customized methods to accurately predict phenotypes in diverse lines of lima bean.
\end{abstract}

Lima bean is an integral crop of the mid-Atlantic region (MAR) in the United States, where Delaware produces over $60 \%$ of the nation's crop for canning and freezing (Tolomeo et al. 2013). The cultivated lima bean is thought to have reached the east coast of the United States via a number of trade routes operated by the Native Americans in pre-Columbian times (Mackie 1943). Lima bean is native to tropical Central and South America, with the Andes thought to be the crop's general center of origin (Fofana et al. 1999; Serrano-Serrano et al. 2010). Biochemical and DNA-based assays have demonstrated that lima bean has been domesticated at least twice, giving rise to the Andean gene pool, characterized by large seeds, and the Mesoamerican gene pools (MI and MII), characterized by small seeds (Debouck et al. 1989; Fofana et al. 2001; Gutiérrez Salgado et al. 1995; Nienhuis et al. 1995; Serrano-Serrano et al. 2012).

Lima bean grown for the vegetable processing industry is most productive in cool, humid weather, which is favorable for pollination and pod set (Kee et al. 1997) but also conducive for the onset of plant diseases. Downy mildew of lima bean, caused by Phytophthora phaseoli Thaxt., is one such disease, first discovered in Hamden, Connecticut in 1889 by Thaxter (Thaxter 1889). The pathogen progressively moved south and, by 1903 , Smith had identified the oomycete as being "injurious" in Delaware and Maryland with yield losses of 25 to $90 \%$ being experienced in Frederick County, Maryland in 1905. Breeding for resistance to $P$. phaseoli was initiated in 1946 by United States Department of Agriculture

Corresponding author: N. M. Donofrio; E-mail address: ndonof@udel.edu

*The $\boldsymbol{e}$-Xtra logo stands for "electronic extra" and indicates that two supplementary figures and three supplementary tables are published online.

http://dx.doi.org/10.1094/PHYTO-02-16-0087-FI

(C) 2016 The American Phytopathological Society
(USDA) researchers, who found resistant accessions of lima bean from different parts of the world (Wester 1961; Wester and Jorgensen 1959). Recurrent adaptation of the pathogen to genetic resistance deployed against it over the years resulted in epidemics in the 1950s and 1960s (Zaumeyer and Wester 1968), with the most recent adaptation of the pathogen to a resistance $(R)$ gene occurring in 2000 , when an estimated production loss of $40 \%$, equal to a farm value loss of $\$ 3,000,000$ was experienced in Delaware (Evans et al. 2007). There are six documented physiological races of $P$. phaseoli (races A through $\mathrm{F}$ ); race $\mathrm{A}$ was the initial race discovered by Thaxter in 1889 , while race $\mathrm{F}$ is currently predominant in the field and has been found in grower fields since the year 2000 (Davidson et al. 2008; Evans et al. 2002).

Breeding for resistance has been a major form of control against downy mildew. A lima bean breeding program was initiated at the University of Delaware in 2004 to develop new cultivars for the MAR, including cultivars with improved resistance. Fungicides play a major role in disease management, their efficiency is improved by concurrent use of disease risk prediction models (Evans et al. 2007). However, mefanoxam-resistant isolates of P. phaseoli have been detected (T. A. Evans, personal communication), elevating the importance of genetic resistance, which is easier to deploy, cheaper, and environmentally friendlier than chemical control. Traditional breeding and selection methods currently in use are generally dependent on favorable weather conditions and multiple crop seasons to develop new cultivars. Marker-assisted selection has been used to speed up selection for traits such as drought resistance, seed quality, and growth habit in other legumes (Kumar et al. 2011; Schneider et al. 1997; Torres et al. 2010). Most of these crops, however, have had substantial research conducted on them, resulting in valuable resources such as sequenced genomes, linkage maps, and characterized genes, none of which are available for lima bean. The advent of high-throughput, 
affordable sequencing technology allows such resources to begin being generated for lima bean.

Genetic analysis of populations using reduced representation sequencing approaches, such as restriction site-associated DNA tagging and genotyping-by-sequencing (GBS), subsample the genome at homologous locations across individuals, allowing the identification of single nucleotide polymorphisms (SNPs) evenly throughout the genome (Catchen et al. 2013; Elshire et al. 2011; Poland et al. 2012). GBS, in particular, has numerous advantages that include more cost efficiency and less labor than other available methods (Poland et al. 2012). The challenge with GBS is in working with organisms that lack a reference genome. While the use of de novo assembly of short sequence reads via software such as STACKS allows circumvention of the need for a reference genome (Catchen et al. 2013), sequence validation is complicated and caveats such as incorporating sequencing artifacts and errors into genotype data must be considered. The availability of statistical methods and resources such as segregating populations with known phenotypes are useful in such instances, allowing the validation of sequence data from GBS analysis (Elshire et al. 2011; Nielsen et al. 2011). Loci identified through methods that utilize GBS can be converted into marker assays such as KASP (Kompetitive allelespecific polymerase chain reaction [PCR]) assays, a uniplex genotyping technology that utilizes allele-specific oligo extension and fluorescence resonance energy transfer (FRET) for signal generation (http://www.lgcgroup.com) (Semagn et al. 2013). This genotyping technology allows markers that have been identified through sequencing to be tested on different individuals at lower costs and with higher throughput (Hiremath et al. 2012). In this paper, we will discuss how GBS was utilized to obtain markers for race $\mathrm{F}$ resistance in lima bean. These markers were then validated and were used to predict resistance to race $\mathrm{F}$ in a recently acquired diverse panel of lima bean accessions, the first time these methods have been used in lima bean breeding.

\section{MATERIALS AND METHODS}

Plant material. An $\mathrm{F}_{2}$ population consisting of 216 individuals was created by self-hybridizing a single individual from a cross between B2C (resistant to race F of P. phaseoli) (Evans et al. 2007) and Bridgeton (susceptible to race $\mathrm{F}$ of $P$. phaseoli). Based on pedigree records, B2C is expected to be at least $80 \%$ similar to Bridgeton (Stavely 1991; Thomas and Fisher 1980). This $F_{2}$ population was utilized to develop DNA markers for resistance to race $\mathrm{F}$ of downy mildew through bulked segregant analysis (Michelmore et al. 1991) using GBS (BSA-GBS). A geographically diverse collection of lima bean was used to validate the predictive ability of BSA-GBS-derived DNA markers for resistance to race F of downy mildew. This lima bean collection, hereafter referred to as the diversity panel, consisted of accessions sourced from The International Center for Tropical Agriculture (CIAT), the USDA, some seed companies, University of California lima bean breeding program, and the University of Delaware lima bean breeding program (Supplementary Table S1). Accessions in the diversity panel originated mainly from the Andean and Mesoamerican regions, the Caribbean Islands, and cultivars developed in the United States. Accessions in the panel also included some landraces from Africa (Nigeria and Zimbabwe), Asia (China and India), and Europe (France). Each individual of the $\mathrm{F}_{2}$ population $(\mathrm{B} 2 \mathrm{C} \times$ Bridgeton) and the diversity panel were grown in 4-in. pots. The first trifoliate leaves were collected, uniquely labeled, frozen, and lyophilized in preparation for DNA extraction.

Inoculum preparation, inoculation, and disease evaluation. Inoculum was maintained and multiplied on Concentrated Fordhook lima bean, which is susceptible to all known races of $P$. phaseoli. Initial inoculum in the form of sporangia and mycelia was obtained from 2-week-old cultures of the $P$. phaseoli race F isolate PhyP18 grown on frozen lima bean agar. The isolate was obtained from the
University of Delaware's plant pathology culture collection. Sporangia of PhyP18 used as inoculum were obtained by weekly inoculations of progressively increasing numbers of 4-day-old Concentrated Fordhook seedlings, using a slurry derived from blending previously infected seedling hypocotyls in distilled water. Inoculated plants were maintained in a digitally controlled dew chamber (Percival 128 Scientific, Inc., Perry, IA) set at $20^{\circ} \mathrm{C}$, relative humidity of $94 \% \pm 1 \%$, and 24-h light with an intensity of $50 \mu \mathrm{mol} \mathrm{m}^{-2} \mathrm{~s}^{-1}$ of photosynthetic active radiation (PAR). Inoculated seedlings showed signs and symptoms of downy mildew after approximately 7 days, upon which they were used as inoculum. This process was repeated until a sufficient amount of inoculum was available to inoculate the $F_{2}$ population planted in the field, using sporangia suspended in distilled water and quantified to $1 \times 10^{3}$ sporangia per milliliter, using a hemocytometer.

Seedlings from the $\mathrm{F}_{2}$ population derived from $\mathrm{B} 2 \mathrm{C} \times$ Bridgeton were transplanted to a research field in Georgetown, Delaware, in a completely randomized design with all plants in a single row and susceptible border rows on either side. One-inch irrigation was provided weekly, using a traveling linear system as required, until the flat pod stage when lima bean is most susceptible to downy mildew. Inoculations with race $\mathrm{F}$ of $P$. phaseoli were performed at the flat pod stage through two inoculations conducted at 10-day intervals in September 2008. Inoculations were conducted using a backpack low-pressure manual pump sprayer (Solo backpack sprayer, model 425, SOLO Incorporated, Newport News, VA) in the early evening, when temperatures were cooler. After inoculation, plants were misted intermittently each night using Micro-Bird misting nozzles (Rain Bird Corp., Glendora, CA) from 6 p.m. to 6 a.m., to increase humidity and a leaf wetness duration of at least $12 \mathrm{~h}$. Individual plants were evaluated for signs and symptoms of downy mildew weekly for a month. Each plant was evaluated for evidence of signs (white fluffy mycelium on shoot tips, pins, and flat pods) and symptoms (red line surrounding signs on pods) of downy mildew. When necessary, confirmation of infection was made by evaluating samples microscopically for sporangia of $P$. phaseoli. Plants that did not develop signs or symptoms of downy mildew were considered resistant. Adjacent susceptible controls (Fordhook) were heavily infected.

BSA using GBS in lima bean. DNA was extracted from young first trifoliate leaf tissue of each individual from the population using the Qiagen DNeasy 96 plant kit (Qiagen, Valencia, CA). DNA was checked for purity, using the NanoDrop ND-1000 spectrophotometer (Thermo Fisher Scientific, Waltham, MA), was quantified using Picogreen (Thermo Fisher Scientific), and was standardized to $25 \mathrm{ng} / \mu \mathrm{l}$. BSA was performed by making separate bulks of the resistant (140 individuals) and the susceptible (61 individuals) plants, using $200 \mathrm{ng}$ of total DNA per individual. Only 201 of the $216 \mathrm{~F}_{2}$ progeny were used in BSA, due to 15 progeny not surviving to the point of inoculation. Individual DNA bulks were then processed through a custom GBS protocol. Two independently formed replicates of the resistant and susceptible bulks and each of the parents were digested with six different pairs of restriction enzymes: PstI/CviQI; Pst I/MseI; SbfI/CviQI; SbfI/MseI; NgoMVI/ CviQI, and NgoMVI/MseI. Restricted DNA samples were ligated with barcoded adaptors (R. J. Wisser, unpublished data) and were then pooled. Proportionate DNA (in nanograms) from the resistant and susceptible bulks was mixed to match the ratio of resistant and susceptible individuals (140:61). Pooled DNA was size-selected to $300 \mathrm{bp}( \pm 30)$ using the BluePippin (Sage Science, Beverley, MA). The size-selected DNA was PCR-amplified, quantified, and sequenced, using 101-bp single end sequencing on the Illumina HiSeq at the Delaware Biotechnology Institute.

Genotype data analysis. The resulting sequence data were processed and analyzed using a custom REDuced REPresentation (redrep) bioinformatics pipeline that wraps open-source tools (primary components: fastqc (Andrews 2010); BWA-MEM (Li 2013); GATK (McKenna et al. 2010, DePristo et al. 2011), and 
custom scripts for processing GBS data (https://github.com/UD$\mathrm{CBCB} / \mathrm{RedRep}$ ). The redrep pipeline for reference-free analysis consists of sequence read quality control (redrep-qc), de novo reference assembly (redrep-cluster), mapping of reads onto the reference (redrep-refmap), and variant calling (redrep-SNPcall). Raw GBS sequence data were processed using redrep-qc to group reads by barcode ID and trim barcode and adaptor sequences from the reads. The next steps in QC were to filter out reads lacking the expected restriction site overhang sequences, short reads ( $<96$ bp), and reads with a mean Phred quality score $<30$. Reduced representation reads from Bridgeton were used to construct the de novo reference, using redrep-cluster, due to its higher read depth and quality compared with B2C. Redrep-cluster is a cluster-based approach that iteratively utilizes the USEARCH cluster small_mem algorithm, defining each cluster by one sequence known as the centroid, or representative, sequence (Edgar 2010). The effect of different clustering identity thresholds of $0.98 \mathrm{id}$ (98\%), $0.99 \mathrm{id}$ $(99 \%)$, and $1.0 \mathrm{id}(100 \%)$ on the redrep reference were compared. Identity thresholds are a parameter in which clusters are based on a percentage similarity to a centroid (representative sequence for which a cluster is based). Sequence reads from the entire library were then mapped against each of the redrep reference genomes and variant identifications were performed.

Filters for read depth $(<12)$, Phred scaled quality scores $(<30)$, and missing genotype data per SNP locus were applied to the variant call data. Further filters were also applied to discard all markers that were not homozygous for the susceptible reference allele in Bridgeton, homozygous for the reference allele in B2C, or heterozygous in both parents, leaving 146 loci. Statistical analyses to identify loci significantly segregating with the race $F$ resistance phenotype were conducted on the post-filter SNPs. The Fisher's exact test $(P<0.01)$ was applied to the data that were not filtered, resulting in 12 loci that were significantly associated with the race F resistance phenotype. These 12 loci were advanced for KASP assay development.

KASP assay design, $F_{2}$ population genotyping, and mapping of the race $\mathbf{F}$ resistance locus. KASP primers were designed (Supplementary Table S2) around the SNPs, using Primer3 primer design software (Koressaar and Remm 2007; Untergasser et al. 2012). Primers were synthesized by Integrated DNA Technologies (Coralville, IA). PCR conditions were optimized for each KASP assay primer and were visualized on a $2 \%$ agarose gel (Supplementary Fig. S1) to ensure optimal thermocycling conditions for each primer, before running KASP assays on the ABI 7900HT fast qPCR instrument (Thermo Fisher Scientific). KASP assays were conducted on the ABI 7900HT fast qPCR instrument, using the high Rox master mix according to manufacturer's recommendations (LGC Genomics, Beverly, MA). SNP assays were performed using each of the pass filter SNPs on each individual of the biparental $F_{2}$ progeny and were scored as being homozygous reference (Bridgeton allele), homozygous nonreference (B2C allele), or heterozygous. The genotype for each of the $\mathrm{F}_{2}$ progeny was scored using the "allelic discrimination" assay module on the Sequence Detection Systems software version 2.3 and then manually, to validate the software's genotype calling. Marker data obtained through KASP assay genotyping were analyzed using the R packages R/qtl version 2.0-4 and Onemap. Onemap was used to determine the recombination fraction between all pairs of markers, using two-point tests. Once recombination fractions had been calculated and tested, markers were arranged into linkage groups and mapping was carried out using the Kosambi mapping function and the seriation algorithm of Onemap to order markers, to estimate the intermarker distances, and to plot a linkage map for the race $\mathrm{F}$ resistance locus (Margarido et al. 2013). Interval mapping to determine the effect of each locus on race $F$ resistance and to estimate
A

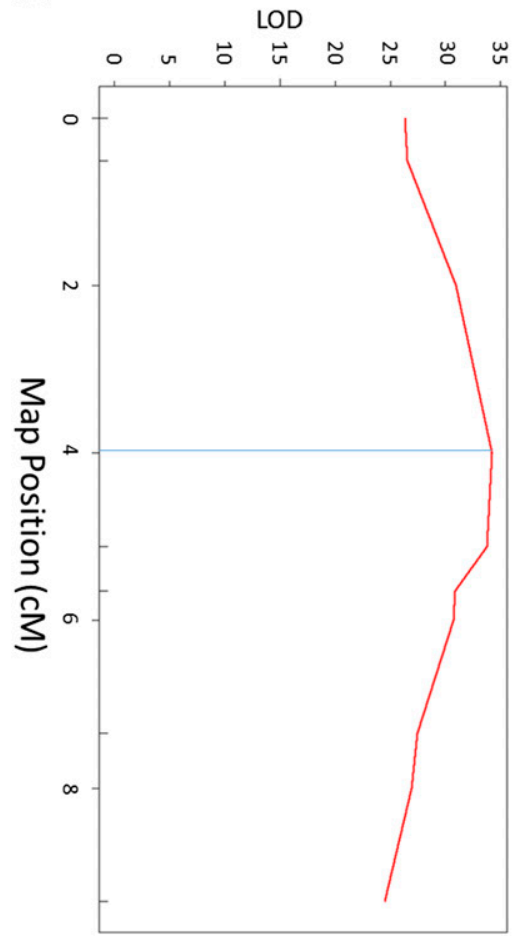

B

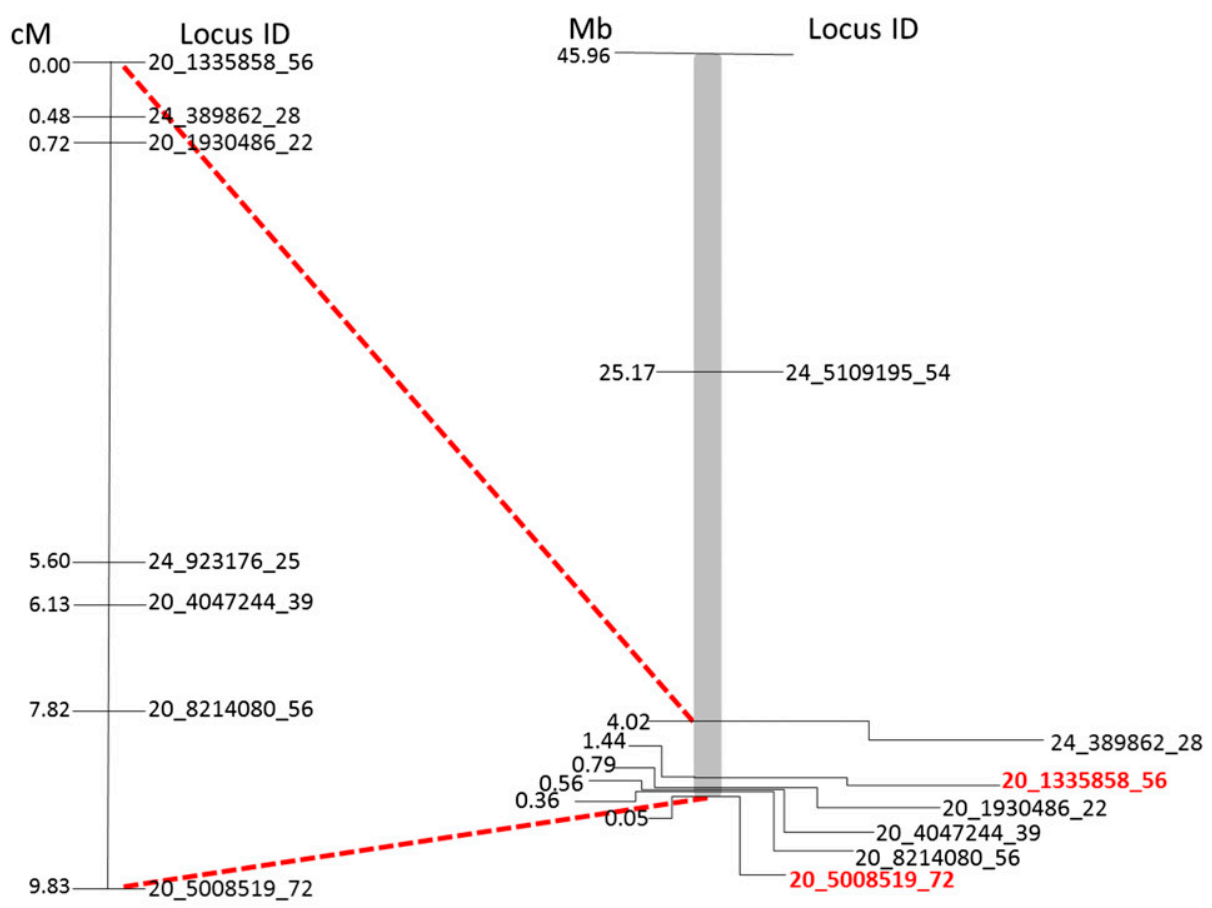

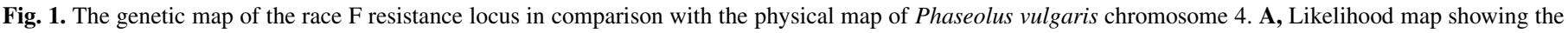

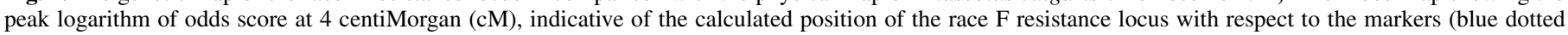

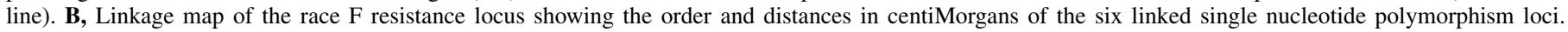

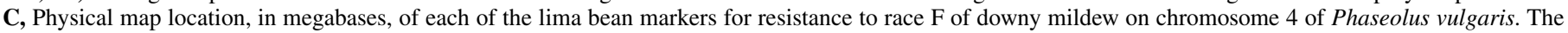

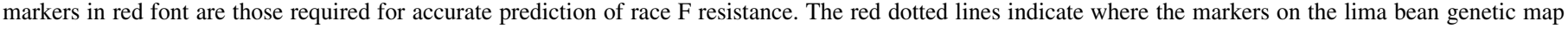
span chromosome 4 of Phaseolus vulgaris. 
the location of the race $\mathrm{F}$ locus was done using Haley-Knott regression in R/qtl (Broman and Sen 2009; Margarido et al. 2013).

Utility and validation of race $F$ resistance molecular markers in a diversity panel. In order to ascertain the utility of markers obtained using BSA-GBS as a race F resistance prediction tool, 256 diverse accessions of lima bean were genotyped, using the KASP assays developed in this study. Six possible multilocus genotypes (MLGs) were identified after genotyping each accession of the

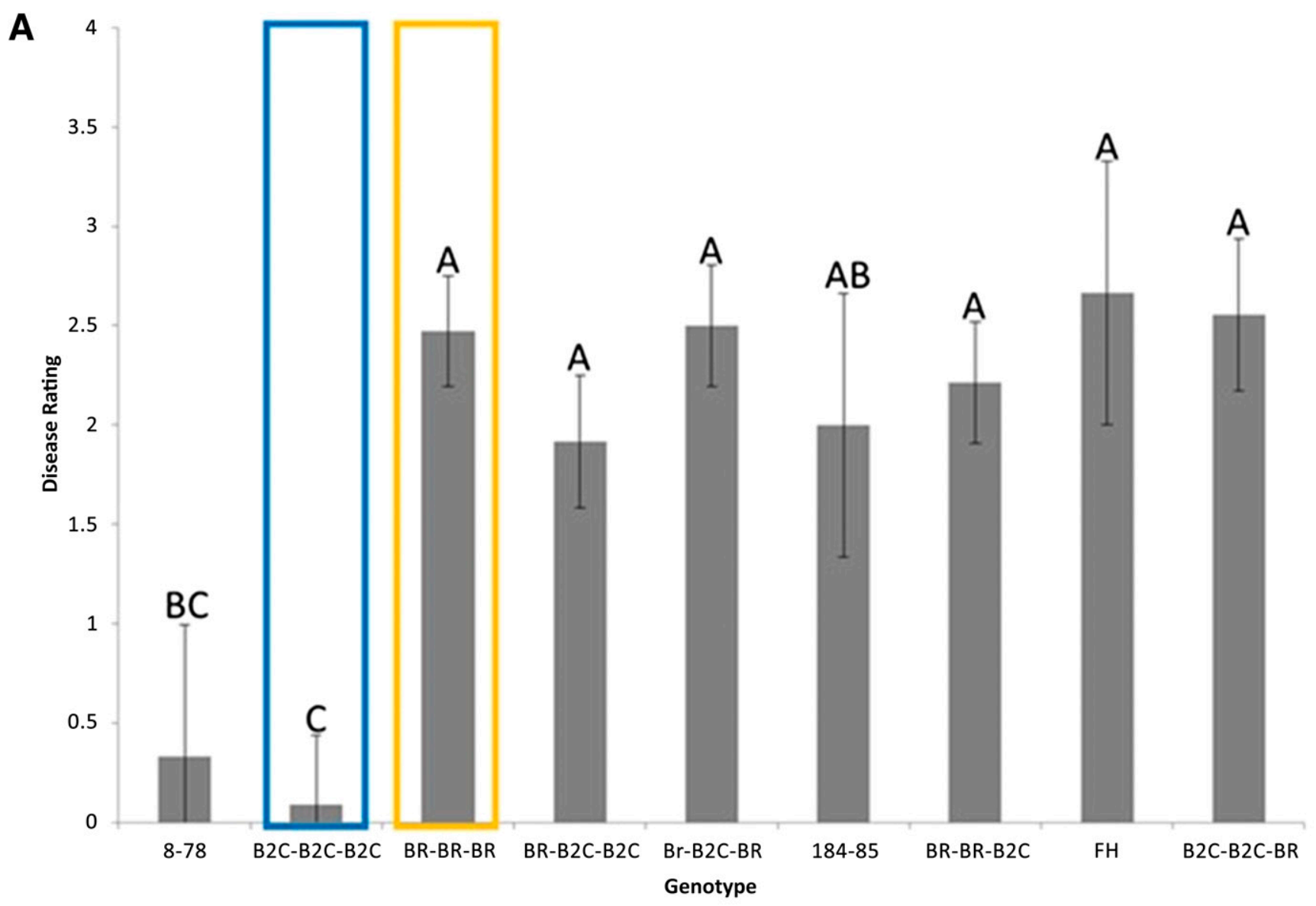

B

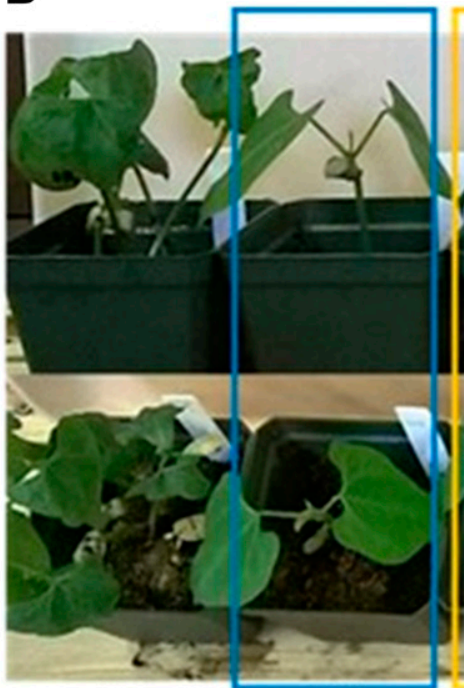

(a) (b)

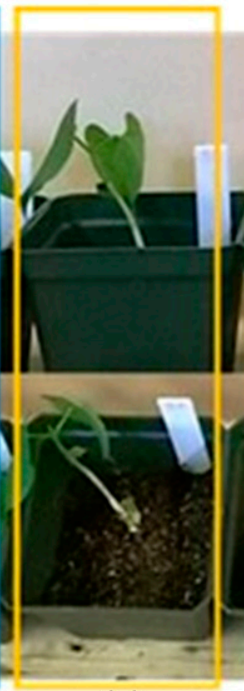

(c)

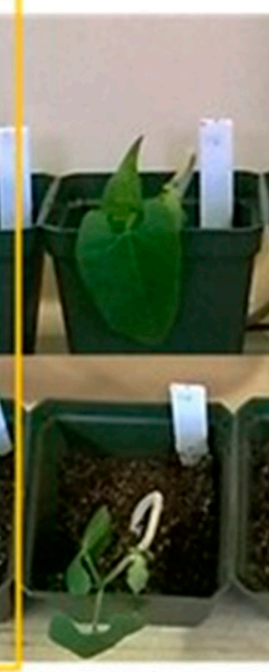

(d)

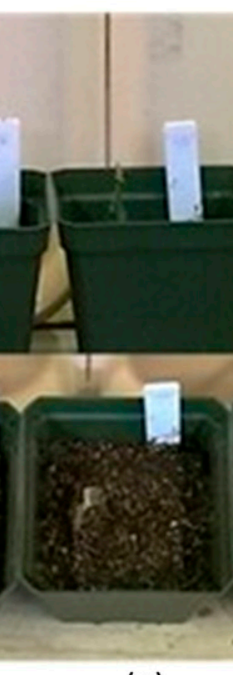

(e)

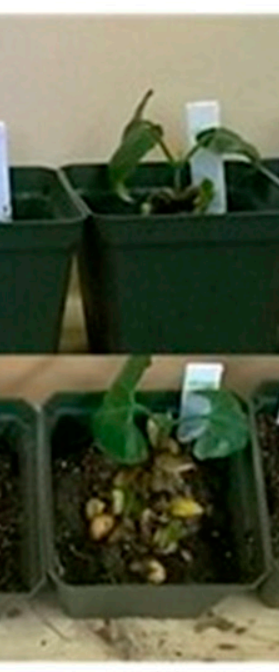

(f)

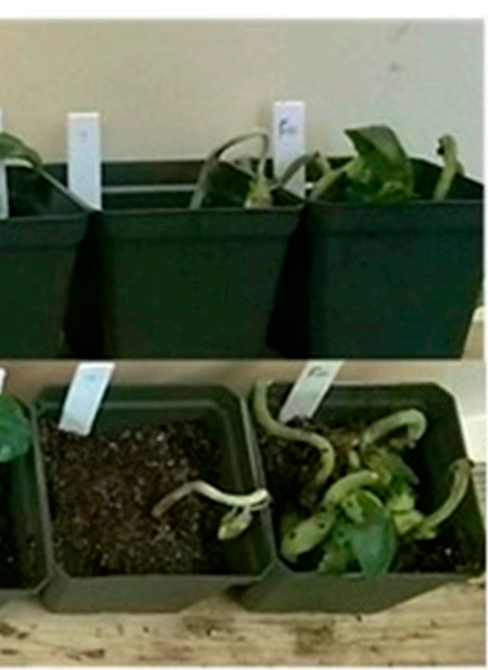

(g)

(h)

Fig. 2. Disease ratings and images of accessions consisting of the different marker haplotypes after inoculation with Phyp18. Haplotypes are made up of single nucleotide polymorphism variants of the markers 20_1335858_56,20_5008519_72, and 20_1930486_22. A, Ratings of infection by Phytophthora phaseoli, using a 0 to 4 rating scale on diversity panel accessions grouped by haplotype. Cultivars 8-78 (race F resistant), $184-85$ (race F susceptible), and Fordhook (universally susceptible) are used as controls. The blue pane shows the B2C-B2C-B2C haplotype, which is accurately predicting race F resistance. The orange panel is the anomaly, where the BR-BR-BR haplotype, overall predicted as susceptible, has one resistant accession (PI 347779). B, Different accessions in different haplotype groups reacted phenotypically to race F inoculation relative to each other. The pots are photographed from a sideways and aerial position from pot (a) to (h). (a), 8-78 from the USA; (b), accession PI 200919 from El Salvador, with haplotype B2C-B2C-B2C; (c), accession PI 347779 (Hopi 13) from the United States, with haplotype BR-BR-BR but containing slow mildewing traits, thus, being able to withstand severe infection; (d),1102-26 from the Dominican Republic, with haplotype BR-B2C-B2C; (e), accession PI 347819 (Willow leaf white) from the United States, with the haplotype BR-B2C-BR; (f), 184-85 from the United States, with a BR-BR-BR haplotype; (g), accession 1102-3A from Haiti, with haplotype BR-BR-B2C; and (h), accession PI 549479 (concentrated Fordhook) from the United States, with the haplotype BR-BR-BR. 
diversity panel. Between three and five individuals from each haplotype group were selected, based on how many individuals were available per MLG. In this case, a haplotype is defined as a group of alleles that are found together. A randomized complete block design with three replicates per accession was used (only three seeds per accession were planted due to limited seed availability). Each seed was planted in a 4-in. pot containing redi earth. The pots were arranged in a randomized complete block design and were watered and allowed to germinate on seedling heat mats. At germination, seed coats were carefully removed from each germinating seed and the emerging hypocotyls were inoculated using the previously described method. Each accession was individually scored, after 5 days in the dew chamber, as being either resistant or susceptible. In order to ascertain if any of the accessions that were being screened contained the "slow mildewing" trait that is also desirable, a rating scale of 0 to 4 was used to rate infections $(0$, no infection; $1,<50 \%$ of the plant covered with mycelia; $2,>50 \%$ of the plant covered with mycelia; $3,<50 \%$ with wilted seedling; 4, >50\% with wilted seedling). Disease rating data were analyzed using analysis of variance. The Dunnett's posthoc analysis was then used to compare each MLG to the susceptible control Concentrated Fordhook in order to ascertain which MLGs were significantly different from the control.

\section{RESULTS}

BSA using GBS for a nonreference organism. Disease evaluations were conducted on the inoculated $\mathrm{F}_{2}$ population planted in the University of Delaware's Research and Education Center, Georgetown research field after approximately 7 days. A total of 140 resistant progeny and 61 susceptible progeny were observed, with 15 progeny failing to survive to the point of inoculation (Supplementary Table S3). The $\chi^{2}$ test showed that segregation of the race $\mathrm{F}$ resistance phenotype followed a 3:1 segregation ratio indicating a dominant resistance phenotype $\left(\chi^{2}=3.066,2\right.$ degrees of freedom, $P<0.05)$. A multiplexed library of different paired enzyme treatments on the BSA samples was used to identify SNPs associated with race $F$ resistance. A total of 47 million raw sequence reads (GeneBank accession PRJNA322045) were analyzed, using the redrep nonref pipeline pathway. Following redrep-qc, $12.5 \%$ of the sequences were filtered. Among the six paired enzyme treatments used for GBS, sequences from PstI and $C v i \mathrm{QI}$ represented $47 \%$ of the total post-QC data. Consequently, this enzyme treatment yielded the greatest number of loci with sufficient read depth for further analysis.

We used three clustering settings in order to determine the threshold that would best result in the retention of polymorphic loci. Genetic expectations were then used as the basis to filter out nonconforming loci and determine an optimal threshold setting (Supplementary Fig. S2). The 1.0 ID (100\% identity) threshold was ultimately used to construct the de novo reference.

Development of molecular markers associated with race F resistance and resistance locus mapping. BSA-GBS-associated SNPs were converted into KASP assays. Conventional PCR showed that eight of the 12 assays amplified. These were used in KASP assays to genotype the individual $\mathrm{F}_{2}$ progeny. Seven of these eight markers mapped onto a single locus spanning $9.83 \mathrm{cM}$, which cosegregated with resistance to race F of downy mildew. The flanking markers most strongly associated with resistance (20_1930486_22 and 24_923176_25) were 3.28 and $1.60 \mathrm{cM}$ away from the inferred location of the resistance gene (Fig. 1A and B).

Validation of the predictive accuracy of molecular markers on a lima bean diversity panel. All eight functional KASP assays were used to genotype the diversity panel consisting of 256 cultivars, landraces, and wild germplasm. Among the United States cultivars were accessions whose race $F$ resistance phenotype was known from previously conducted field inoculations (Maffei 15, resistant; Cypress, C-elite, and Concentrated Fordhook, susceptible) (Ernest et al. 2011).
Resistance to downy mildew in these accessions with known response to race $\mathrm{F}$ was accurately predicted, using three of the seven KASP assays (20_1335858_56,20_5008519_72, and 20_1930486_22). The diversity panel contained six of the nine theoretically possible haplotypes for these markers, and the B2C-resistance haplotype was rare (found in four of 256 accessions) in the collection.

Inoculations with race $\mathrm{F}$ were performed on a subset of accessions selected based on their haplotypes to further assess the predictability of the three-marker haplotype. All of the tested accessions that were homozygous for the B2C haplotype were resistant, whereas $85 \%$ of the accessions heterozygous or homozygous for the Bridgeton allele were susceptible (Fig. 2). The B2C-B2C-B2C haplotype (Fig. 2A, highlighted by the blue box,) was precise in predicting resistance to race $\mathrm{F}$, however, only two of these markers, after a complete screening of the diversity panel, are necessary for accurate prediction of race $F$ resistance. Four accessions of the 256 in the diversity panel were characterized by this haplotype, including Maffei 15 (M15), Sussex, PI200919 (Cojutepeque, El Salvador), and PI200924 (Jalopa, Guatemala). Screening results using PhyP18 on accessions containing the other haplotypes showed that only two of the markers were necessary to accurately predict resistance (20_1335858_56 and 20_5008519_72). However, there were resistant individuals that contained alternative haplotypes, including BR-B2C-B2C for PI 310627 (Chaparota from Guatemala), BR-BR-B2C for PI 256816 (Frijol tierno from Ecuador), and BR-BR-BR for PI 256405 (Chilipuca from El Salvador).

Collinearity of lima bean race $\mathrm{F}$ resistance map with Phaseolus vulgaris. All eight markers were mapped to the genome of a closely related species, Phaseolus vulgaris (Bonifácio et al. 2012; Schmutz et al. 2014). Seven of the eight KASP markers were found to span $24.85 \mathrm{Mb}$ on chromosome 4 of common bean (Fig. 1C). The genome space between those markers found on the lima bean linkage map (six of the seven markers spanning a 4-Mb locus on chromosome 4 of Phaseolus vulgaris) consisted of an $R$ gene-dense region containing 110 locus tags (http://www.ncbi.nlm.nih.gov/genome/ proteins/380?genome_assembly_id=48590\&gi=593705291), with 38 annotated as being $R$-gene related (Phytozome; https://phytozome.jgi. doe.gov/) (Goodstein et al. 2012). The seventh marker on the lima bean linkage map maps to chromosome 7 of Phaseolus vulgaris. Furthermore, among the markers that were collinear on common bean and were accurate in predicting race $\mathrm{F}$ resistance (20_1335858_56 and 20_5008519_72, which are $1.39 \mathrm{Mb}$ apart), we identified 21 locus tags containing eight classes of resistance proteins in this region of chromosome 4 of Phaseolus vulgaris (Table 1; Li et al. 2012).

\section{DISCUSSION}

Epidemics produced by race $\mathrm{F}$ of $P$. phaseoli are a threat to Delaware's status of leading lima bean sales to the vegetable processing industry in the MAR and, indeed, to the entire United States. The utility of GBS in lima bean using a nonreference approach was optimized and demonstrated an efficient and high-throughput method for genotyping lima bean and effectively characterizing traits of interest. The use of KASP assays developed from BSA-GBS markers allowed the mapping of a race $F$ resistance locus and the KASP assays effectively identified downy mildew resistant lima bean accessions from genetically diverse plant material. The most important outcome of this method was in the ability of the markers found using the reference-free redrep pipeline to identify race F-resistant lima bean accessions from our diversity panel. The validation of this method opens avenues for further research, allowing for dissection of the genetic architecture of traits of importance in lima bean, such as disease resistance, heat tolerance, growth habit, and cyanide content.

The diversity panel is a resource that is not only useful for validating the approach we used to identify race F resistance loci but can also be useful in the identification of other important traits, including alternative race $F$ resistance loci, through phenotyping the 
entire diversity panel. Likely forms of alternative race $\mathrm{F}$ resistance were, indeed, observed, as three accessions in the diversity panel were found to be resistant to race $\mathrm{F}$ in our phenotype screen but did not contain the predictive haplotype for resistance. This indicates that additional race $F$ resistance loci may be present in other genome locations. These may be in the form of as-yet-unknown resistance genes or quantitative trait loci (QTL). The former could lead to $R$-gene pyramiding, a proven method of increasing durability of resistance in commercial cultivars toward pathogens in a number of crops (Pedersen and Leath 1988). Pyramiding has been demonstrated to increase efficacy against infection and increase durability of resistance, using both major resistance genes and genes conferring partial resistance (Fukuoka et al. 2015; Huang et al. 1997; Li et al. 1999). Alternative forms of resistance, such as QTL, can allow the deployment of multiple resistance genes against $P$. phaseoli in one cultivar, slowing down pathogen adaptation and reducing yield losses due to downy mildew. QTL confer quantitative resistance but can contribute to durable resistance and confer broad-spectrum or non-race specific resistance, providing an additive effect to overall resistance (Fukuoka et al. 2015). The "slow mildewing" trait observed in the accession PI 347779 (Hopi 13 from Arizona, with BR-BR-BR haplotype [Fig. 2B, highlighted by the orange boarder]) is likely to be the effect of QTL, which can be in the form of defeated major genes, such as the $\mathrm{Xa} 4^{T}$ gene in rice, contributing a residual resistance effect against pathogens ( $\mathrm{Li}$ et al. 1999). Slow mildewing has been found to be durable in wheat against Blumeria graminis $\mathrm{f}$. sp. tritici, due to its quantitative nature, and also as an effective means of minimizing disease rates in the field through reduced sporulation and secondary inoculum production (Shaner 1973). Rigorous phenotypic screening of the diversity panel, coupled with the GBS approach taken here, allows the possibility of either resistance pyramiding by targeting these multiple traits and introgressing them into one cultivar or discovering the loci underlying the slow-mildewing phenotype, either of which could lead to more durable resistance. We are currently using the methodology from this study to identify the QTL responsible for the slow-mildewing trait for the subsequent development of improved commercial cultivars.

The loci discovered in this research were mapped to the genome of sister species Phaseolus vulgaris (common bean) and were found to be collinear to the lima bean race $\mathrm{F}$ resistance map. The approximately $4-\mathrm{Mb}$ region spanned by six of the seven loci from the lima bean linkage map consisted of an $R$ gene-enriched region. Additionally, Phytozome was used to conduct an analysis of the Phaseolus vulgaris genome in the approximate region between the loci accurately predicting race $F$ resistance $(1.39 \mathrm{Mb})$, showing the presence of 172 locus tags, with 21 of these being associated with resistance proteins such as coiled-coil nucleotide-binding-site leucine-rich repeats and resistance-related transporters (Table 1) (Goodstein et al. 2012). Eight classes of these resistance-related proteins were found to reside in these 21 locus tags (Table 1). This observation using the syntenic relationship between the two species

TABLE 1. Genome region between the markers accurately predicting race $\mathrm{F}$ resistance are collinear with a resistance gene-dense region in Phaseolus vulgaris

\begin{tabular}{lc}
\hline Resistance protein class $^{\mathrm{a}}$ & Number of appearances \\
\hline CC-NBS-LRR class & 10 \\
TIR-NBS-LRR class & 8 \\
NB-ARC domain & 41 \\
LRR and NB-ARC domain & 21 \\
RPP13 & 48 \\
HopZ-activated resistance & 18 \\
RGA & 32 \\
Putative disease resistance & 13
\end{tabular}

${ }^{a} \mathrm{CC}=$ coiled coil, $\mathrm{NBS}=$ nucleotide binding site, $\mathrm{LRR}=$ leucine-rich repeat, $\mathrm{TIR}=$ toll interleukin 1 receptor, $\mathrm{RGA}=$ resistance gene analog. of bean will allow a more targeted approach to identifying the one or more causal $R$ genes for downy mildew resistance. Indeed, collinearity between species has facilitated identification of markers linked to resistance genes, as in the case of the wheat resistance gene Sr39 and rice (Niu et al. 2011).

Overall, this work establishes a platform that will allow for future dissection of the genetic architecture of other important traits of lima bean, including important but more complex traits, like slow mildewing and abiotic stresses such as heat tolerance, which are important concerns to regional growers (T. A. Evans, personal communication). The development and validation of our method using a tractable genetic experimental population opens up the possibility of in-depth studies of diverse accessions of lima bean using these DNA library preparations and customized bioinformatic pipelines.

\section{ACKNOWLEDGMENTS}

This material is based upon work that is supported by the National Institute of Food and Agriculture, U.S. Department of Agriculture under award number 2012-51181-19776 and the Delaware Department of Agriculture. Expertise and computational infrastructure provided by the University of Delaware Bioinformatics Core Facility was made possible through support from Delaware IDeA Network of Biomedical Research (NIGMS P20 GM103446), the State of Delaware, and the Delaware Biotechnology Institute. We also acknowledge S. Kunjeti for carrying out culture maintenance and inoculum preparations among other duties that allowed this project to be successful.

\section{LITERATURE CITED}

Andrews, S. 2010. FastQC: A quality control tool for high throughput sequence data. Babraham Institute, Cambridge. Available online at: http:// www.bioinformatics.babraham.ac.uk/projects/fastqc

Bonifácio, E. M., Fonsêca, A., Almeida, C., dos Santos, K. G. B., and Pedrosa-Harand, A. 2012. Comparative cytogenetic mapping between the lima bean (Phaseolus lunatus L.) and the common bean (P. vulgaris L.). Theor. Appl. Genet. 124:1513-1520.

Broman, K. W., and Sen, S. 2009. A guide to QTL mapping with R/qtl. Springer, New York.

Catchen, J., Hohenlohe, P., Bassham, S., Amores, A., and Cresko, W. 2013. Stacks: An analysis tool set for population genomics. Mol. Ecol. 22: 3124-3140.

Davidson, C. R., Evans, T. A., Mulrooney, R. P., Gregory, N. F., Carroll, R. B., and O'Neill, N. R. 2008. Lima bean downy mildew epiphytotics caused by new physiological races of Phytophthora phaseoli 2008. Plant Dis. 92: 670-674.

Debouck, D. G., Maquet, A., and Poesso, C. E. 1989. Biochemical evidence for two different gene pools in Lima bean, Phaseolus lunatus L. Annu. Rep. Bean Improv. Coop. 32:58-59.

DePristo, M., Banks, E., Poplin, R., Garimella, K., Maguire, J., Hartl, C., Philippakis, A., del Angel, G., Rivas, M. A., Hanna, M., McKenna, A., Fennell, T., Kernytsky, A., Sivachenko, A., Cibulskis, K., Gabriel, S., Altshuler, D., and Daly, M. 2011. A framework for variation discovery and genotyping using next-generation DNA sequencing data. Nat. Genet. 43: 491-498.

Edgar, R. C. 2010. Search and clustering orders of magnitude faster than BLAST. Bioinformatics 26:2460-2461.

Elshire, R. J., Glaubitz, J. C., Sun, Q., Poland, J. A., Kawamoto, K., Buckler, E. S., and Mitchell, S. E. 2011. A robust, simple genotyping-by-sequencing (GBS) approach for high diversity species. PLoS One 6:e19379.

Ernest, E. G., Evans, T. A., and Gregory, N. F. 2011. An investigation of the sources of resistance to lima bean downy mildew (Phytophthora phaseoli) races $\mathrm{E}$ and $\mathrm{F}$, and identification of Fordhook lima bean cultivars with downy mildew resistance. Annu. Rep. Bean Improv. Coop. 54:98-99.

Evans, T. A., Davidson, C. R., Dominiak, J. D., Mulrooney, R. P., Carroll, R. B., and Antonius, S. H. 2002. Two new races of Phytophthora phaseoli from lima bean in Delaware. Plant Dis. 86:813.

Evans, T. A., Mulrooney, R. P., Gregory, N. F., and Kee, E. 2007. Lima bean downy mildew: Impact, etiology, and management strategies for Delaware and the mid-Atlantic region, U.S. Plant Dis. 91:128-135.

Fofana, B., Baudoin, J. P., Vekemans, X., Debouck, D. G., and du Jardin, P. 1999. Molecular evidence for an Andean origin and a secondary gene pool for the lima bean (Phaseolus lunatus L.) using chloroplast DNA. Theor. Appl. Genet. 98:202-212. 
Fofana, B., du Jardin, P., and Baudoin, J. P. 2001. Genetic diversity in the lima bean (Phaseolus lunatus L.) as revealed by chloroplast DNA (cpDNA) variations. Genet. Resour. Crop Evol. 48:437-445.

Fukuoka, S., Saka, N., Mizukami, Y., Koga, H., Yamanouchi, U., Yoshiako, Y., Hayashi, N., Ebana, K., Mizobachi, R., and Yano, M. 2015. Gene pyramiding enhances durable blast disease resistance in rice. Sci. Rep. 5:7773.

Goodstein, D. M., Shu, S., Howson, R., Neupane, R., Hayes, R. D., Faz, J., Mitros, T., Dirks, W., Hellsten, U., Putnam, N., and Rokhsar, D. S. 2012. Phytozome: A comparative platform for green plant genomics. Nucleic Acids Res. 40:D1178-D1186.

Gutiérrez Salgado, A., Gepts, P., and Debouck, D. G. 1995. Evidence for two gene pools of the lima bean, Phaseolus lunatus L., in the Americas. Genet. Resour. Crop Evol. 42:15-28.

Hiremath, P. J., Kumar, A., Penmetsa, R. V., Farmer, A., Schlueter, J. A., Chamarthi, S. K., Whaley, A. M., Carrasquilla-Garcia, N., Gaur, P. M., Upadhaya, H. D., Kavi Kishor, P. B., Shah, T. M., Cook, D. R., and Varshney, R. V. 2012. Large scale development of cost-effective SNP marker assays for diversity assessment and genetic mapping in chickpea and comparative mapping in legumes. Plant Biotechnol. J. 10:716-732.

Huang, N., Angeles, E. R., Domingo, J., Magpantay, G., Singh, S., Zhang, G., Kumaravadivel, N., Bennet, J., and Khush, G. S. 1997. Pyramiding of bacterial blight resistance genes in rice: Marker-assisted selection using RFLP and PCR. Theor. Appl. Genet. 95:313-320.

Kee, E., Glancey, J. L., and Wooten, T. L. 1997. The lima bean: A vegetable crop for processing. HortTechnology 7:119-128.

Koressaar, T., and Remm, M. 2007. Enhancements and modifications of primer design program Primer3. Bioinformatics 23:1289-1291.

Kumar, J., Choudhary, A. K., Solanki, R. K., and Pratap, A. 2011. Towards marker-assisted selection in pulses: A review. Plant Breed. 130:297-313.

Li, H. 2013. Aligning sequence reads, clone sequences and assembly contigs with BWA-MEM. arXiv:1303.3997 [q-bio.GN].

Li, J., Dai, Z., Liu, T., and Zhao, P. X. 2012. LegumeIP: An integrative database for comparative genomics and transcriptomics of model legumes. Nucleic Acids Res. 40:D1221-D1229.

Li, Z. K., Luo, L. J., Mei, H. W., Paterson, A. H., Zhao, X. H., Zhong, D. B., Wang, Y. P., Yu, X. Q., Zhu, L., Tabien, R., Stansel, J. W., and Ying, C. S. 1999. A "defeated" rice resistance gene acts as a QTL against a virulent strain of Xanthomonas oryzae pv. oryzae. Mol. Gen. Genet. 261:58-63.

Mackie, W. W. 1943. Origin, dispersal and variability of the lima bean, Phaseolus lunatus. Hilgardia 15:1-29.

Margarido, G. R. A., Mollinari, M., and Garcia, A. A. F. 2013. OneMap tutorial: Software for constructing genetic maps in experimental crosses: Full-sib, RILs, F2 and back-crosses. https://cran.r-project.org/web/packages/onemap/ vignettes/Tutorial_Onemap_reduced_version.pdf. Accessed 3 Dec 2015.

McKenna, A., Hanna, M., Banks, E., Sivachenko, A., Cibulskis, K., Kernytsky, A., Garimella, K., Altshuler, D., Gabriel, S., Daly, M., and DePristo, M. A. 2010. The Genome Analysis Toolkit: A MapReduce framework for analyzing next-generation DNA sequencing data. Genome Res. 20:1297-303.

Michelmore, R. W., Paran, I., and Kesseli, R. V. 1991. Identification of markers linked to disease-resistance genes by bulked segregant analysis: A rapid method to detect markers in specific genomic regions by using segregating populations. Proc. Natl. Acad. Sci. USA 88:9828-9832.

Nielsen, R., Paul, J. S., Albrechtsen, A., and Song, Y. 2011. Genotype and SNP calling from next-generation sequencing data. Nat. Genet. 12:443-451.

Nienhuis, J., Skroch, P., and Dos Santos, J. B. 1995. Genetic relationships among cultivars and landraces of Lima bean (Phaseolus lunatus L.) as measured by RAPD markers. J. Am. Soc. Hortic. Sci. 2:300-306.

Niu, Z., Klindworth, D. L., Friesen, T. L., Chao, S., Jin, Y., Cai, X., and Xu, S. S. 2011. Targeted introgression of a wheat stem rust resistance gene by DNA marker assisted chromosome engineering. Genetics 187:1011-1021.
Pedersen, W. L., and Leath, S. 1988. Pyramiding major genes for resistance to maintain residual effects. Annu. Rev. Phytopathol. 26:369-378.

Poland, J. A., Brown, P. J., Sorrells, M. E., and Jannink, J.-L. 2012. Development of High-Density Genetic Maps for Barley and Wheat Using a Novel Two-Enzyme Genotyping-by-Sequencing Approach. PLoS One 7:e32253.

Schmutz, J. A., McClean, P. E., Mamidi, S., Wu, G. A., Cannon, S. B., Grimwood, J., Jenkins, J., Shu, S., Song, Q., Chavarro, C., Torres-Torres, M., Geffroy, V., Moghaddam, S. M., Gao, D., Abernathy, B., Barry, K., Blair, M., Brick, M. A., Chovatia, M., Gepts, P., Goodstein, D. M., Gonzales, M., Hellsten, U., Hyten, D. L., Jia, G., Kelly, J. D., Kudrna, D., Lee, R., Richard, M. M., Miklas, P. N., Osorno, J. M., Rodrigues, J., Thareau, V., Urrea, C. A., Wang, M., Yu, Y., Zhang, M., Wing, R. A., Cregan, P. B., Rokhsar, D. S., and Jackson, S. A. 2014. A reference genome for common bean and genome-wide analysis of dual domestications. Nat. Genet. 46:707-713.

Schneider, K. A., Brothers, M. E., and Kelly, J. D. 1997. Marker-assisted selection to improve drought resistance in common bean. Crop Sci. 37: 51-60.

Semagn, K., Babu, R., Hearne, S., and Olsen, M. 2013. Single nucleotide polymorphism genotyping using Kompetitive Allele Specific PCR (KASP): Overview of the technology and its application in crop improvement. Mol. Breed. 33:1-14.

Serrano-Serrano, M. L., Andueza-Noh, R. H., Martínez-Castillo, J., Debouck, D. G., and Chacón, M. I. 2012. Evolution and domestication of lima bean (Phaseolus lunatus L.) in Mexico: Evidence from ribosomal DNA. Crop Sci. 52:1698-1712.

Serrano-Serrano, M. L., Hernandez-Torres, J., Castillo-Villamizar, G., Debouck, D. G., and Chacón, M. I. 2010. Gene pools in wild lima bean (Phaseolus lunatus L.) from the Americas: Evidences for an Andean origin and past migrations. Mol. Phylogenet. Evol. 54:76-87.

Shaner, G. 1973. Evaluation of slow-mildewing resistance of Knox wheat in the field. Phytopathology 63:867-872.

Stavely, J. R. 1991. Lima bean (Phaseolus lunatus L.) development at Beltsville. Annu. Rep. Bean Improv. Coop. 34:155-156.

Thaxter, R. 1889. Mildew of Lima Beans. (Phytophthora phaseoli Thaxt.). Annu. Rep. Conn. Agric. Exp. Stn. 1890:167-171.

Thomas, C. A., and Fisher, V. J. 1980. Registration of B2C lima bean germplasm. Crop Sci. 520:553.

Tolomeo, V., Krug, K., Flohr, D., Holm, S., Jeutong, R., Johnson, J., Kugel, B., Letterman, J., McDonnell, J., Olmstead, K., Schwedler, L. and Van Court, J. 2013. California Vegetable Review. United States Department of Agriculture National Agricultural Statistics Service 34.

Torres, A. M., Avila, C. M., Gutierrez, N., Palomino, C., Moreno, M. T., and Cubero, J. J. 2010. Marker-assisted selection in faba bean (Vicia faba L.). Field Crops Res. 115:243-252.

Untergasser, A., Cutcutache, I., Koressaar, T., Ye, J., Faircloth, B. C., Remm, M., and Rozen, S. G. 2012. Primer3 - new capabilities and interfaces. Nucleic Acids Res. 40:e115.

Wester, R. E. 1961. Resistance to downy Mildew of lima bean. Page 16 in: Annual Report of the Bean Improvement Cooperative and National Dry Bean Council Research Conference, USDA.

Wester, R. E., and Jorgensen, H. 1959. A New Race of Downy Mildew of Lima Bean. Pages 18-19 in: Annual Report of the Bean Improvement Cooperative and National Dry Bean Council Research Conference, USDA.

Zaumeyer, W. J., and Wester, R. E. 1968. The Rapid Development and Spread of Strain B of Phytophthora phaseoli in 1967. Pages 51-52 in: Annual Report of the Bean Improvement Cooperative and National Dry Bean Council Research Conference, USDA. 